Check for updates

Cite this: RSC Adv., 2018, 8, 38667

\title{
Charge-induced electromechanical actuation of Mo- and W-dichalcogenide monolayers
}

\begin{abstract}
Vuong Van Thanh, (D) *a Nguyen Tuan Hung (D) *b and Do Van Truong ${ }^{\text {cd }}$
Using first-principle density functional calculations, we investigate electromechanical properties of twodimensional $\mathrm{MX}_{2}(\mathrm{M}=\mathrm{Mo}, \mathrm{W} ; \mathrm{X}=\mathrm{S}, \mathrm{Se}, \mathrm{Te})$ monolayers with the $1 \mathrm{H}$ and $1 \mathrm{~T}$ structures as a function of charge doping for both electron and hole doping. We find that by increasing the atomic number, $Z_{X}$, of $X$ atoms $\left(Z_{\mathrm{S}}<Z_{\mathrm{Se}}<Z_{\mathrm{Te}}\right)$, the work density per cycle of the $M X_{2}$ monolayers are increased and decreased for the $1 \mathrm{H}$ and $1 \mathrm{~T}$ structures, respectively. On the other hand, the work density per cycle of the $W_{2}$ monolayers are higher than that of the $\mathrm{MoX}_{2}$ monolayers for both the $1 \mathrm{H}$ and $1 \mathrm{~T}$ structures. Therefore, $W \mathrm{We}_{2}$ and $\mathrm{WS}_{2}$ monolayers for the $1 \mathrm{H}$ and $1 \mathrm{~T}$ structures, respectively, have the best electromechanical performances in the $\mathrm{MX}_{2}$ compounds. In addition, the $\mathrm{MX}_{2}$ monolayers show a reversible strain up to $3 \%$, which is higher than that of graphene $(\sim 1 \%)$. Our results provide an important insight into the electromechanical properties of the $\mathrm{MX}_{2}$ monolayers, which are useful for artificial muscles applications.
\end{abstract}

Received 5th October 2018

Accepted 13th November 2018

DOI: $10.1039 / \mathrm{c} 8 \mathrm{ra0} 8248 \mathrm{k}$

rsc.li/rsc-advances

Transition-metal dichalcogenides (TMDs) such as $\mathrm{MX}_{2}(\mathrm{M}=$

\section{Introduction}

Artificial muscles or electromechanical actuation based on twodimensional (2D) materials have become attractive recently because of its excellent properties such as larger surface doping, mechanical flexibility and thermal/chemical stability. ${ }^{1-5}$ For example, Xie et al. ${ }^{6}$ reported the superior strain response of a $2 \mathrm{D}$ graphene actuator, which has a strain approaching to $0.85 \%$, exceeding that of the best-known CNT-based actuators. On the other hand, Ge et al. ${ }^{7}$ showed that an actuator based on the graphene oxide film is able to lift more than eight times its own weight. Very recently, Lu et al. ${ }^{8}$ reported a 2D graphdiyne-based electromechanical actuator with a high strain of up to $6.03 \%$, and its energy density $\left(11.5 \mathrm{~kJ} \mathrm{~m}^{-3}\right)$ is comparable to that of mammalian skeletal muscle $\left(\sim 8 \mathrm{~kJ} \mathrm{~m}^{-3}\right)$. However, the 2D artificial muscles based on graphene, graphene oxide, and graphdiyne have still been limited in the large-scale applications such as biomedicine and rehabilitation devices because these actuator materials are expensive and difficult to synthesize. Therefore, finding new 2D actuator materials with low manufacturing cost and large strain amplitude for the artificial muscles should be necessary.

${ }^{a}$ Department of Design of Machinery and Robot, School of Mechanical Engineering, Hanoi University of Science and Technology, Hanoi, Vietnam. E-mail: thanh. vuongvan@hust.edu.vn

${ }^{b}$ Department of Physics, Tohoku University, Sendai 980-8578, Japan. E-mail: nguyen@ flex.phys.tohoku.ac.jp

'Department of Mechatronics, School of Mechanical Engineering, Hanoi University of Science and Technology, Hanoi, Vietnam

${ }^{d}$ International Institute for Computational Science and Engineering, Hanoi University of Science and Technology, Hanoi, Vietnam
$\mathrm{Mo}, \mathrm{W} ; \mathrm{X}=\mathrm{S}, \mathrm{Se}, \mathrm{Te}$ ) have similar layered structures as graphene., ${ }^{90}$ The chemically exfoliated $\mathrm{MoS}_{2}$ nanosheets are synthesized by organo-lithium chemistry. By characteristics such as large-area two-dimensional flakes, thermodynamically stable and high quality, the $2 \mathrm{D} \mathrm{MoS}_{2}$ nanosheets have very attractived for artificial muscles on a large scale applications. Recently, Acerce et al. ${ }^{11}$ showed a significant performance on the electromechanical actuation of the $2 \mathrm{D} \mathrm{MoS}$ nanosheets by using the intercalation reactions. They reported that the mechanical stress, strain, and work density of the $\mathrm{MoS}_{2}$ nanosheets reach about $17 \mathrm{MPa}, 0.8 \%$, and $81 \mathrm{~kJ} \mathrm{~m}^{-3}$, respectively. However, their study has limited to the $\mathrm{MoS}_{2}$ nanosheets with only $1 \mathrm{~T}$ structure. By using the density functional theory (DFT) calculations, Hung et al. ${ }^{12}$ investigated the actuator performance of the $\mathrm{MoS}_{2}$ monolayer with the $1 \mathrm{H}, 1 \mathrm{~T}$ and $1 \mathrm{~T}^{\prime}$ structures. They pointed out that the actuator performance of the $\mathrm{MoS}_{2}$ monolayers with the $1 \mathrm{~T}$ and $1 \mathrm{~T}^{\prime}$ structures are better than that of the $1 \mathrm{H}$ structure. This because the interplay between the actuation strain and Young's modulus of the $\mathrm{MoS}_{2}$ monolayer with difference structures. Both experiment and theory ${ }^{11,12}$ only focused of the $\mathrm{MoS}_{2}$ monolayer actuator, while the actuator properties of other TMDs are not fully explored yet, except for some limited experiments. ${ }^{13}$ In this sense, a systematic theoretical design of many TMDs materials could be the first step to suggest possible structures suitable as highest electromechanical actuators. Moreover, TMDs have been demonstrated that the $1 \mathrm{H}$ structure is more dominant than the $1 \mathrm{~T}$ and $1 \mathrm{~T}^{\prime}$ structure in many experiments ${ }^{14-16}$ due to their low energy formation. Therefore, it is an important to find which TMD among $\mathrm{MX}_{2}$ compounds has a high actuator performance with the $1 \mathrm{H}$ structure. 
In this paper, we use the first-principles calculations within DFT to calculate the electromechanical properties of the $\mathrm{MX}_{2}$ monolayers with a variety of $M(M=M o, W)$ and $X(X=S, S e, T e)$ atoms as a function of charge doping for both electron and hole doping. As the main highlight of this paper, we summarizes all the periodic trends strictly obeyed by our data including the Young's modulus, work density per cycle and actuator stress for both $1 \mathrm{H}$ and $1 \mathrm{~T}$ structures of the $\mathrm{MX}_{2}$ monolayers. Our results show that $\mathrm{WTe}_{2}$ and $\mathrm{WS}_{2}$ monolayers with the $1 \mathrm{H}$ and $1 \mathrm{~T}$ structures, respectively, have the best electromechanical performances in the $\mathrm{MX}_{2}$ compounds at heavy electron doping.

\section{Methodology}

The density-functional theory are performed to calculate the electromechanical actuator of the $\mathrm{MX}_{2}$ monolayers using the Quantum ESPRESSO package. ${ }^{17}$ The pseudopotentials from the Standard Solid-State Pseudopotentials library are used. ${ }^{18}$ The exchange-correlation energy is evaluated by the general gradient approximation using the Perdew-Burke-Ernzerhof ${ }^{19}$ function. The cutoff energy of plane wave is set at $60 \mathrm{Ry}$, and $16 \times 16 \times 1$ $k$-point mesh of Monkhorst-Pack scheme is used for Brillouin zone integrations. ${ }^{20}$

The atomic structures of the $\mathrm{MX}_{2}$ monolayers with the $1 \mathrm{H}$ and $1 \mathrm{~T}$ structures are shown in Fig. 1. The atomic positions and cell vectors of the $\mathrm{MX}_{2}$ monolayers are fully relaxed to obtain optimized atomic configurations by using the BroydenFletcher-Goldfarb-Shanno minimization method ${ }^{21-24}$ until all the Hellmann Feynman forces and all components of the stress are less than 0.0005 Ry/a.u. and $0.05 \mathrm{GPa}$, respectively. The periodic boundary condition is applied in all models, a vacuum space of $30 \AA$ in the direction perpendicular to the monolayer $(z$ direction) is used in order to avoid virtual interactions between layers.

The geometry optimization is then performed for each charge doping from -0.1 to +0.1 electron per atom (e/atom) to consider the electromechanical actuation of the $\mathrm{MX}_{2}$ monolayers. The electron (hole) doping is simulated by adding (removing) electrons to the unit cell with a uniform charge (a) $1 \mathrm{H}-\mathrm{MX}_{2}$
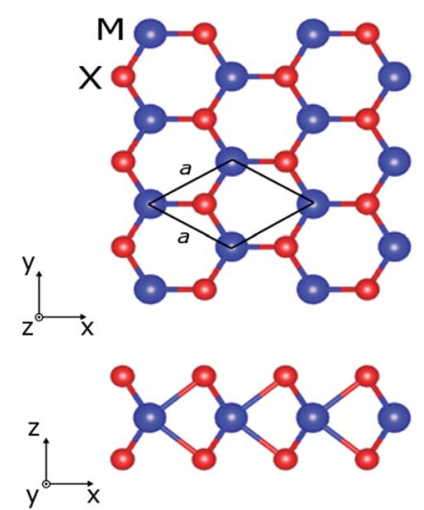

(b) $1 \mathrm{~T}-\mathrm{MX}_{2}$

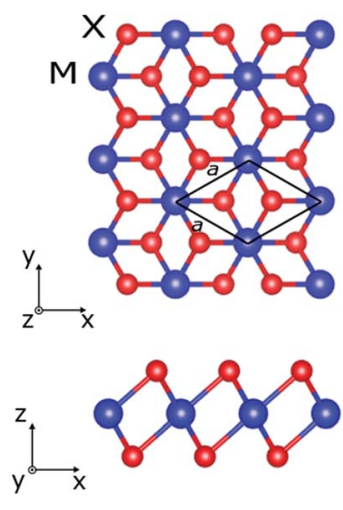

Fig. 1 Top and side view of the $M X_{2}$ monolayers with the $1 \mathrm{H}(\mathrm{a})$ and $1 \mathrm{~T}$ structures (b), respectively. background..$^{25,26}$ The elastic constants $C_{i j}$ of $\mathrm{MX}_{2}$ monolayers are estimated by using the Themo-pw code. ${ }^{27}$ Since the values of $C_{i j}$ are related to the equivalent volume of the unit cell, the calculated $C_{i j}$ must be rescaled by $h / d_{0}$, where $h$ is the length of the cell along $z$ axis and $d_{0}$ is the effective layer thickness of the $\mathrm{MX}_{2}$ monolayers $\left(d_{0}=6.145 \AA\right) .{ }^{12}$ The Young's modulus, $Y$, and the Poisson's ratio, $\nu$, of the $\mathrm{MX}_{2}$ monolayers can be obtained from the following equation as ${ }^{12}$

$$
Y=\frac{C_{11}^{2}-C_{12}^{2}}{C_{11}} ; \nu=\frac{C_{12}}{C_{11}}
$$

The power of electromechanical actuators is characterized by the stress generated, $\sigma=Y \varepsilon$ where actuator strain $\varepsilon$ is defined as $\Delta a / a_{0}$, where $a_{0}$ is the lattice constant at geometry optimization for neutral case, and $\Delta a$ is increment (or decrement) of $a_{0}$ after the charge doping has been applied. The performance of electromechanical actuators is characterized by the work density per cycle, which is often expressed in terms of stored energy density $W_{\mathrm{s}} \cdot{ }^{12}$ The formula for the work density per cycle is given by

$$
W_{\mathrm{s}}=\frac{1}{2} Y \varepsilon^{2} .
$$

\section{Results and discussion}

In Table 1, we show the optimized lattice parameters of the $\mathrm{MX}_{2}$ monolayers, in which the lattice parameters of the $\mathrm{MoX}_{2}$ and $\mathrm{WX}_{2}$ monolayers have quite similar values. The lattice constants of the $\mathrm{MX}_{2}$ monolayers increase with increasing of atomic number of $\mathrm{M}, Z_{\mathrm{M}}\left(Z_{\mathrm{Mo}}<Z_{\mathrm{W}}\right)$, and $\mathrm{X}, Z_{\mathrm{X}}\left(Z_{\mathrm{S}}<Z_{\mathrm{Se}}<Z_{\mathrm{Te}}\right)$, atoms. Our calculated results are in good agreement with previous theoretical results, ${ }^{9,28}$ indicating that the present calculations are reasonable and reliable. In addition the total energy of the $\mathrm{MX}_{2}$ monolayers with the $1 \mathrm{H}$ structure $\left(1 \mathrm{H}-\mathrm{MX}_{2}\right)$ are smaller than that of the $1 \mathrm{~T}$ structure $\left(1 \mathrm{~T}-\mathrm{MX}_{2}\right)$. Therefore, the $\mathrm{MX}_{2}$ monolayers with $1 \mathrm{H}$ structure are more stable than $1 \mathrm{~T}$ structure. In many experiments, the $1 \mathrm{H}$ structure also was found to be

Table 1 Lattice constant $a_{0}(\AA)$, total energy $E_{\text {tot }}($ Ry), elastic constants $C_{i j}(\mathrm{GPa})$, Young's modulus $Y(\mathrm{GPa})$ and Poisson's ratio $\nu$ of the $1 \mathrm{H}$ - and $1 \mathrm{~T}-\mathrm{MX}_{2}$ monolayers

\begin{tabular}{lllllllll}
\hline $\mathrm{MX}_{2}$ & $a_{0}$ & $E_{\text {tot }}$ & $C_{11}$ & $C_{22}$ & $C_{12}$ & $C_{66}$ & \multicolumn{1}{l}{$\nu$} \\
\hline $1 \mathrm{H}-\mathrm{MoS}_{2}$ & 3.19 & -190.798 & 207 & 207 & 42 & 83 & 197 & 0.200 \\
$1 \mathrm{H}-\mathrm{MoSe}_{2}$ & 3.31 & -224.510 & 173 & 173 & 32 & 71 & 167 & 0.180 \\
$1 \mathrm{H}-\mathrm{MoTe}_{2}$ & 3.55 & -190.204 & 129 & 129 & 41 & 44 & 116 & 0.320 \\
$1 \mathrm{~T}-\mathrm{MoS}_{2}$ & 3.18 & -190.739 & 169 & 169 & -4.0 & 86 & 167 & -0.020 \\
$1 \mathrm{~T}-\mathrm{MoSe}_{2}$ & 3.28 & -224.462 & 166 & 166 & -3.2 & 85 & 166 & -0.019 \\
$1 \mathrm{~T}-\mathrm{MoTe}_{2}$ & 3.53 & -190.169 & 140 & 140 & -0.39 & 70 & 140 & -0.003 \\
$1 \mathrm{H}-\mathrm{WS}_{2}$ & 3.19 & -597.594 & 230 & 230 & 43 & 94 & 222 & 0.185 \\
$1 \mathrm{H}-\mathrm{WSe}_{2}$ & 3.32 & -631.292 & 193 & 193 & 31 & 81 & 188 & 0.160 \\
$1 \mathrm{H}-\mathrm{WTe}_{2}$ & 3.56 & -596.969 & 140 & 140 & 27 & 57 & 135 & 0.190 \\
$1 \mathrm{~T}-\mathrm{WS}_{2}$ & 3.20 & -597.530 & 171 & 171 & -2.2 & 87 & 171 & -0.013 \\
$1 \mathrm{~T}-\mathrm{WSe}_{2}$ & 3.29 & -631.238 & 159 & 159 & -9.3 & 84 & 159 & -0.060 \\
$1 \mathrm{~T}-\mathrm{WTe}_{2}$ & 3.54 & -596.931 & 136 & 136 & -12 & 73 & 135 & -0.086
\end{tabular}


abundantly in sample. ${ }^{29,30} \mathrm{We}$ also note that $\mathrm{MoTe}_{2}$ and $\mathrm{WTe}_{2}$ monolayers might not have ideal $1 \mathrm{~T}$ phase. They form the distorted $1 \mathrm{~T}$ structure, namely $1 \mathrm{~T}^{\prime}$ structure. ${ }^{31}$ Since the $\mathrm{MX}_{2}$ monolayers with $1 \mathrm{~T}^{\prime}$ structure exhibit an anisotropic behavior, ${ }^{12,31}$ in present study, we only focus on the isotropic $1 \mathrm{H}-$ and $1 \mathrm{~T}-\mathrm{MX}_{2}$ monolayers.

To calculate the actuator response of the $\mathrm{MX}_{2}$ monolayers, we firstly check the mechanical moduli at the neutral condition case $(q=0)$. The values of the elastic constants $C_{i j}$, Young's modulus $Y$ and Poisson's ratio $\nu$ of the $\mathrm{MX}_{2}$ monolayers are listed in Table 1. $Y$ of the $\mathrm{MX}_{2}$ monolayers decreases with increasing $Z_{\mathrm{X}}$. On the other hand, by increasing $Z_{\mathrm{M}}, Y$ is increased and decreased for the $1 \mathrm{H}$ and $1 \mathrm{~T}$ structures, respectively, expect that $Y$ of the $1 \mathrm{~T}-\mathrm{MoS}_{2}$ monolayer is smaller than that of the $1 \mathrm{~T}-\mathrm{WS}_{2}$ monolayer. Therefore, the $\mathrm{WS}_{2}$ monolayer shows the stiffest materials in the $\mathrm{MX}_{2}$ compounds with $Y=$ $222 \mathrm{GPa}$ and $Y=171 \mathrm{GPa}$ for the $1 \mathrm{H}$ and $1 \mathrm{~T}$ structures, respectively. In addition, the Poisson's ratio is also an important mechanical properties. Our results show that $\nu$ of the $1 \mathrm{H}$ structures is positive, while $\nu$ of the $1 \mathrm{~T}$ structures is negative. It is known that the negative Poisson's ratio are also found in other 2D materials such as in black phosphorus $(\nu=-0.5),{ }^{32}$ single-layer graphene $\operatorname{ribbons}(\nu=-1.51),{ }^{33}$ and $\delta$-phosphorene $(\nu=-0.267) \cdot{ }^{34}$ The $2 \mathrm{D}$ materials with native Poisson's ratio could have useful applications, for example, as vanes for aircraft gas turbine engines, sponges, and fasteners. ${ }^{35}$

Although the atomic structures of the $\mathrm{MX}_{2}$ monolayers has been optimized, they do not guarantee a dynamical stability under the charge doping. Therefore, we analyze the generic elastic stability conditions for the $\mathrm{MX}_{2}$ structures at each charge doping level. In particular, in the $2 \mathrm{D}$ hexagonal structure, the necessary and sufficient conditions of stability are $C_{11}>\left|C_{12}\right|>0$ and $C_{66}>$ $0 .{ }^{36}$ In Fig. 2(a) and (b), we show the elastic constants $\left(C_{11}, C_{12}\right.$ and $C_{66}$ ) of the $\mathrm{MX}_{2}$ monolayers as a function of charge doping $q$ ranging from -0.1 to 0.1 e/atom for the $1 \mathrm{H}$ and $1 \mathrm{~T}$ structures, respectively. Our results reveal that $C_{11}>\left|C_{12}\right|>0$ and $C_{66}>0$ for both all the $1 \mathrm{H}$ and $1 \mathrm{~T}$ structures. Therefore, the $\mathrm{MX}_{2}$ monolayers show a stable structure under both electron and hole doping cases. After revealing the stable configurations, the electromechanical properties of the $\mathrm{MX}_{2}$ monolayers are investigated.

In Fig. 3(a) and (b), we show the Young's modulus $Y$ of the $1 \mathrm{H}$ and $1 \mathrm{~T}-\mathrm{MX}_{2}$ monolayers, respectively, as a function of the charge doping $(q \neq 0)$. For the $1 \mathrm{H}-\mathrm{MX}_{2}$ monolayers, $Y$ decreases for both the electron $(q<0)$ and hole $(q>0)$ dopings, except that $Y$ of the $\mathrm{WS}_{2}$ monolayer is approximately constant for the hole doping case, as shown in Fig. 3(a). For the $1 \mathrm{~T}-\mathrm{MX}_{2}$ monolayers, $Y$ decreases and increases for the electron and hole dopings, respectively, except that $Y$ of the $\mathrm{MoTe}_{2}$ decreases for both electron and hole dopings, as shown in Fig. 3(b). We note that since $C_{12} \ll C_{11}$ (see Fig. 2), the Young's modulus in eqn (1) can rewritten as $Y \sim C_{11}$ for both $1 \mathrm{H}$ - and $1 \mathrm{~T}-\mathrm{MX}_{2}$ monolayers. Therefore, the behaviours of $Y$ are similar to $C_{11}$ under the electron and hole doping cases. The results obtained show that the maximum $Y$ of the $\mathrm{MX}_{2}$ monolayers are $222 \mathrm{GPa}$ and $197 \mathrm{GPa}$ for the $1 \mathrm{H}-\mathrm{WS}_{2}$ and $1 \mathrm{~T}-\mathrm{MoS}_{2}$ monolayers, respectively, at $q=0.1 \mathrm{e} /$ atom. $Y$ of the $\mathrm{MX}_{2}$ monolayers is thus comparable to that of
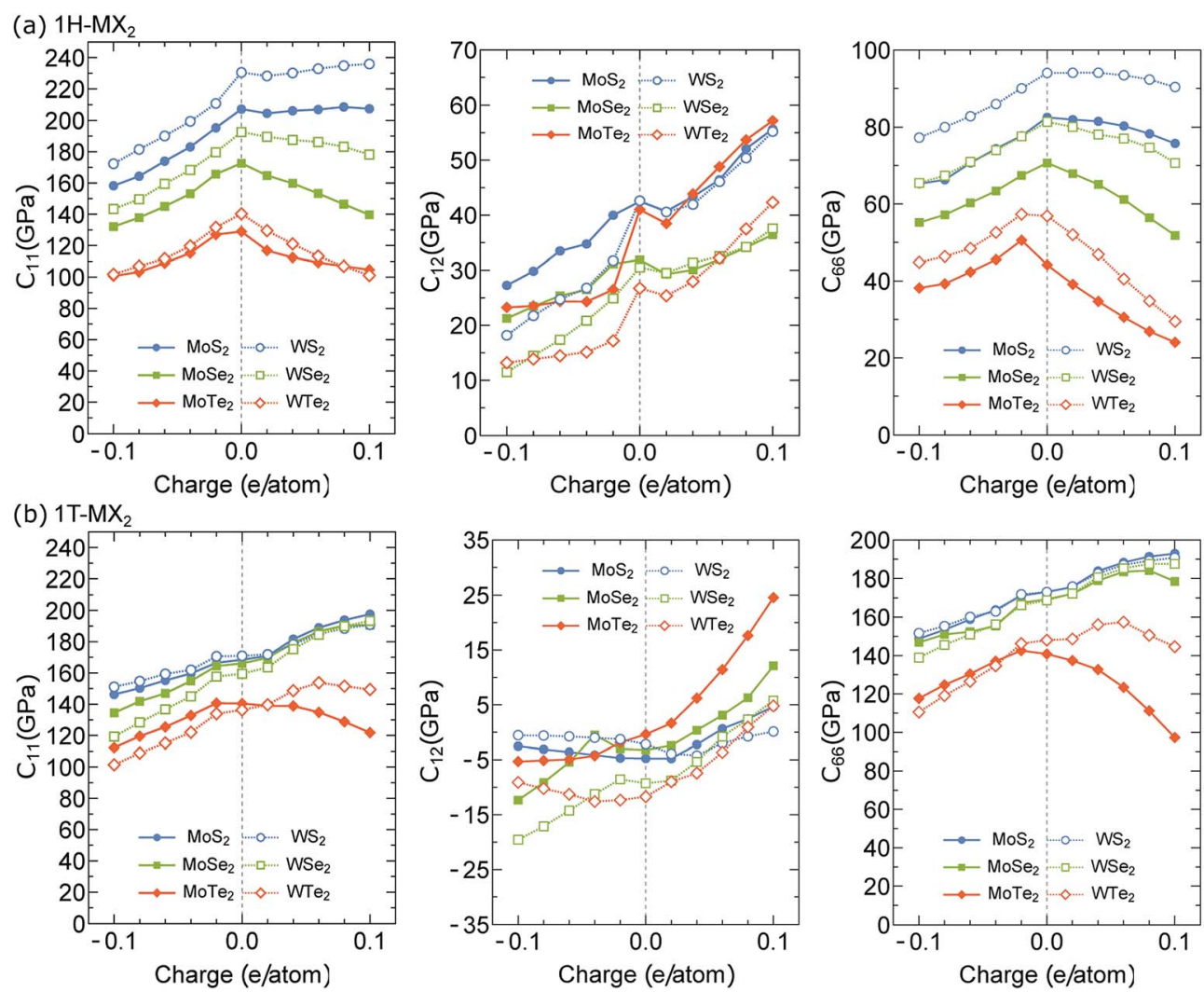

Fig. 2 Elastic constants $C_{11}, C_{12}$ and $C_{66}$ of the (a) $1 \mathrm{H}-M X_{2}$ and (b) $1 \mathrm{~T}-M X_{2}$ monolayers plotted as function of charge (electron and hole) doping per atom. 

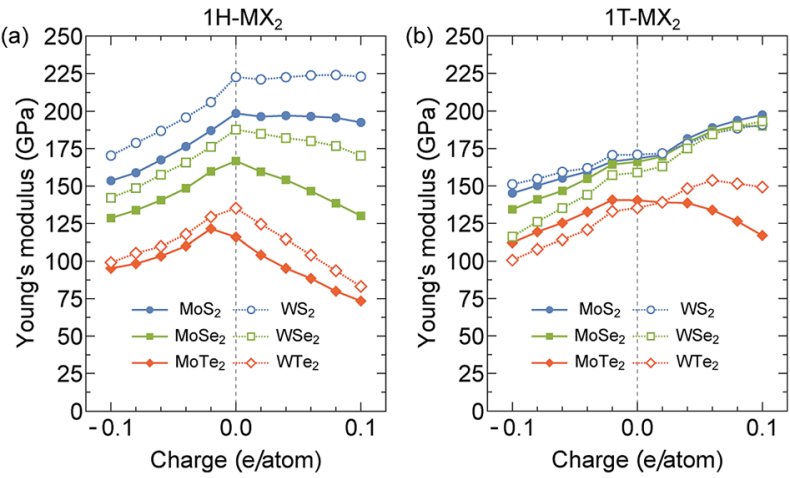

Fig. 3 Young's modulus of the (a) $1 \mathrm{H}-\mathrm{MX}_{2}$ and (b) $1 \mathrm{~T}-\mathrm{MX} \mathrm{X}_{2}$ monolayers plotted as a function of charge doping per atom.

the stainless steel $(192 \mathrm{GPa}) .^{37}$ It notes that the materials with high $Y$ value can generate large force per unit area.

In Fig. 4(a) and (b), we show the actuator strain $\varepsilon$ of the $1 \mathrm{H}^{-}$ and $1 \mathrm{~T}-\mathrm{MX}_{2}$ monolayers as a function of charge doping $q$ ranging from -0.1 to $0.1 \mathrm{e} /$ atom. In the neutral case $(q=0)$, we obtain $\varepsilon=0$. For the charge doping case, $\varepsilon$ is approximately a linear function of $q$ for electron doping $(q<0)$, while it is nonlinear function of $q$ for hole doping $(q>0)$. As shown in Fig. 4(a), the absolute values of $\varepsilon$ of the $1 \mathrm{H}-\mathrm{MX}_{2}$ monolayers mainly depend on X atoms, in which $\mathrm{MTe}_{2}>\mathrm{MSe}_{2}>\mathrm{MS}_{2}$ for both electron and hole doping cases. In contrast, the absolute values
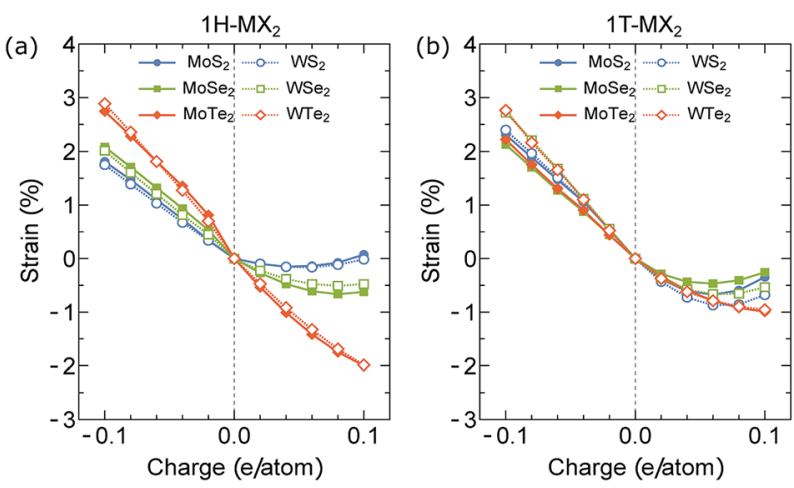

Fig. 4 Strain as function of charge doping per atom of monolayer (a) $1 \mathrm{H}-\mathrm{MX}_{2}$, (b) $1 \mathrm{~T}-\mathrm{MX}_{2}$. of $\varepsilon$ of the 1T-MX $\mathrm{M}_{2}$ monolayers mainly depend on $\mathrm{M}$ atoms ( $\mathrm{WX}_{2}$ $>\mathrm{MoX}_{2}$ ), as shown in Fig. 4(b). For the $\mathrm{MoS}_{2}$ monolayer, the strain of the $1 \mathrm{~T}$ structure $(2.25 \%)$ is higher than that of the $1 \mathrm{H}$ structure $(1.78 \%)$ at $q=-0.1$ e/atom. The $\mathrm{MoS}_{2}$ monolayer with the $1 \mathrm{~T}$ structure is thus predicted high actuation performance, which is consistent with previous results. ${ }^{12}$ As shown in Fig. 4(a) and (b), unlike the $\mathrm{MS}_{2}$ and $\mathrm{MSe}_{2}$ monolayers, the strain of the $\mathrm{MTe}_{2}$ monolayers $\left(\mathrm{MoTe}_{2}\right.$ and $\left.\mathrm{WTe}{ }_{2}\right)$ with the $1 \mathrm{H}$ structure are higher than that with the $1 \mathrm{~T}$ structures. The highest $\varepsilon$ is found in the $\mathrm{MoTe}_{2}(2.74 \%)$ and $\mathrm{WTe}_{2}(2.9 \%)$ monolayers with the $1 \mathrm{H}$ structure at $q=-0.1 \mathrm{e} /$ atom, respectively, which is higher than that of carbon nanotubes and graphene $(\sim 1 \%)^{6,625,38}$ Since the $1 \mathrm{H}$ structure is dominates in the TMDs structures, the $\mathrm{MoTe}_{2}$ and $\mathrm{WTe}_{2}$ monolayers may be the ideal choice for the artificial muscles. In order to understand these behaviors of the actuator strain, the bond lengths between $\mathrm{M}$ and $\mathrm{X}$ atoms are investigated at different charge doping level, as shown in Fig. 5 . In the neutral case $(q=0)$, the $\mathrm{M}(\mathrm{Mo}, \mathrm{W})-\mathrm{Te}$ bond lengths of $1 \mathrm{H}$ - and $1 \mathrm{~T}-\mathrm{MX}_{2}$ are higher than the $\mathrm{M}-\mathrm{Se}$ and $\mathrm{M}-\mathrm{S}$ bond lengths, respectively. For the electron doping, the $\mathrm{M}-\mathrm{X}$ bond lengths increase linearly by increasing $q(q<0)$, while they increase nonlinearly for the hole doping $(q>0)$. As shown in Fig. 5(a)-(c), the electron doping largely change the $\mathrm{M}-\mathrm{X}$ bond lengths compared with the hole doping. We note that the electron doping will "pull down" many interlayer bands in the TMD's band structure, while hole doping do not. ${ }^{12}$ Such a phenomenon might contribute to the higher strain actuators by the electron doping rather than the hole doping.

In Fig. 6(a) and (b), we show the actuator stress of the $\mathrm{MX}_{2}$ monolayers as a function of charge doping for the $1 \mathrm{H}$ and $1 \mathrm{~T}$ structures, respectively. In the neutral case $(q=0)$, we obtain $\sigma=$ 0 because $\varepsilon=0$. For the electron doping $(q<0)$, the stress of the $\mathrm{MX}_{2}$ monolayers with both the $1 \mathrm{H}$ and $1 \mathrm{~T}$ structures increases with increasing $|q|$. The highest stresses are found in the $\mathrm{WS}_{2}$ monolayers for both the $1 \mathrm{H}(\sigma=3 \mathrm{GPa})$ and $1 \mathrm{~T}$ structures $(\sigma=3.6 \mathrm{GPa})$ due to their highest Young's modulus as shown in Fig. 3. For the hole doping $(q>0)$, the highest stress of the $\mathrm{MX}_{2}$ monolayers with the $1 \mathrm{H}$ structure is found in the $\mathrm{WTe}_{2}$ monolayers with $\sigma=$ $-1.65 \mathrm{GPa}$ at $q=0.1 \mathrm{e} / \mathrm{atom}$, while it is found in $\mathrm{WS}_{2}$ monolayers with $1 \mathrm{~T}$ structures $(\sigma=-1.62 \mathrm{GPa}$ at $q=0.1 \mathrm{e} /$ atom).

Finally, we investigate the performance of the electromechanical actuators of the $\mathrm{MX}_{2}$ monolayers. In Fig. 7(a) and (b),
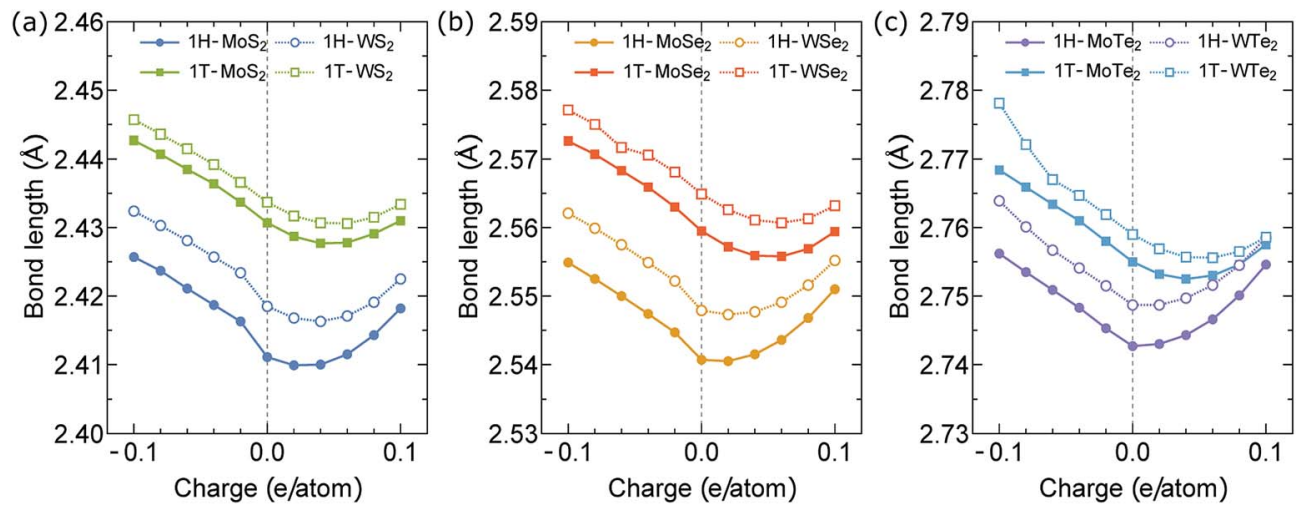

Fig. $5 \mathrm{M}-\mathrm{X}$ bond lengths as a function of the charge doping per atom (a) $\mathrm{MS}_{2}$, (b) $\mathrm{MSe}_{2}$ and (c) $M T e_{2}$. 

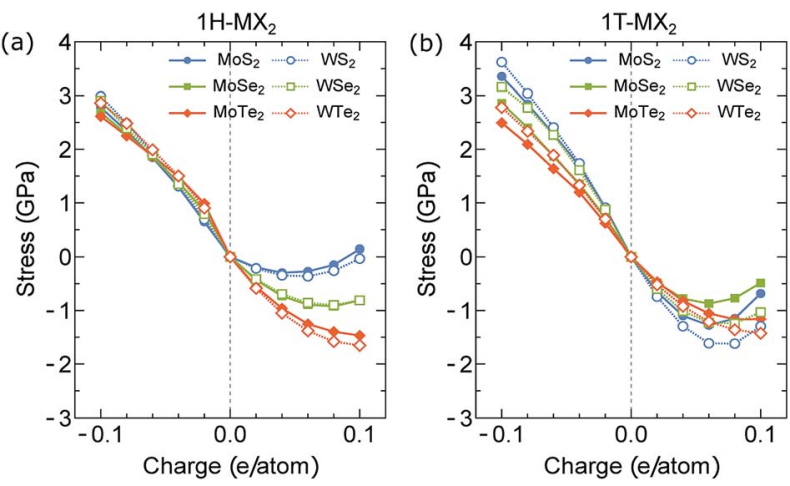

Fig. 6 Stress generated by (a) $1 \mathrm{H}-M X_{2}$ and (b) $1 T-M X_{2}$ monolayers plotted as function of charge doping per atom.
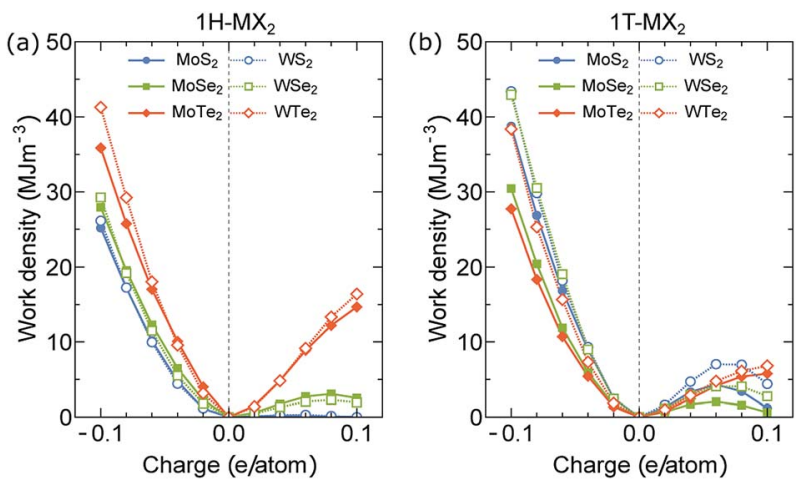

Fig. 7 Work density per cycle of the (a) $1 \mathrm{H}-M X_{2}$ and (b) $1 T-M X_{2}$ monolayers plotted as function of charge (electron and hole) doping per atom.

we show the work density per cycle $W_{\mathrm{S}}$ of the $1 \mathrm{H}$ - and $1 \mathrm{~T}-\mathrm{MX}_{2}$ monolayers as a function of charge doping, respectively, in which the $W_{\mathrm{s}}$ is determined by eqn (2). For the electron doping $(q<0), W_{\mathrm{s}}$ of the $\mathrm{MX}_{2}$ monolayer with both $1 \mathrm{H}$ and $1 \mathrm{~T}$ structures increases with increasing $|q|$. In the meantime, $W_{\mathrm{s}}$ is increased and decreased with increasing $Z_{\mathrm{X}}\left(Z_{\mathrm{S}}<Z_{\mathrm{Se}}<Z_{\mathrm{Te}}\right)$ for the $1 \mathrm{H}$ and $1 \mathrm{~T}$ structures, respectively. On the other hand, $W_{\mathrm{s}}$ of the $\mathrm{MoX}_{2}$ (a)

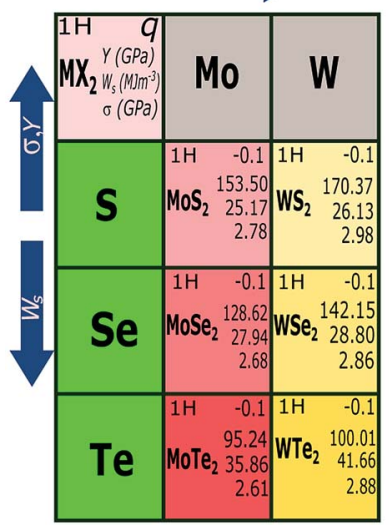

(b)

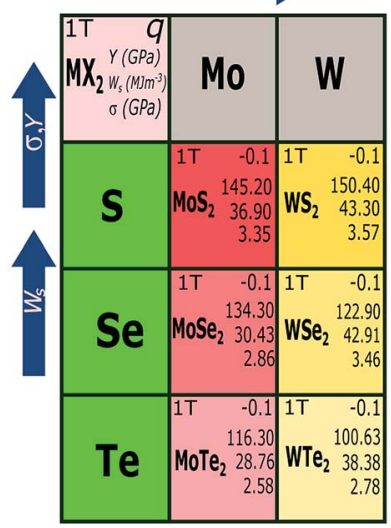

Fig. 8 Trends in work density per cycle, stress and Young's modulus of (a) the $1 \mathrm{H}-\mathrm{MX}_{2}$ and (b) $1 \mathrm{~T}-\mathrm{MX}_{2}$ monolayers for the electron doping at $q$ $=-0.1$ e/atom monolayers is smaller than that of the $\mathrm{WX}_{2}$ monolayers for both $1 \mathrm{H}$ and $1 \mathrm{~T}$ structures. Therefore, the highest $W_{\mathrm{s}}$ is found in the $\mathrm{WTe}_{2}\left(41.66 \mathrm{MJ} \mathrm{m}^{-3}\right)$ and $\mathrm{WS}_{2}\left(43.30 \mathrm{MJ} \mathrm{m}^{-3}\right)$ monolayers with the $1 \mathrm{H}$ and $1 \mathrm{~T}$ structures at $q=-0.1 \mathrm{e} /$ atom, respectively. These work density per cycle are more than 1000 times that of skeleton muscle $\left(\sim 0.04 \mathrm{MJ} \mathrm{m}^{-3}\right)^{39}$ and 7 times that of Au-Pt alloys ( $\sim 6 \mathrm{MJ}$ $\left.\mathrm{m}^{-3}\right)^{40,41}$ due to their high Young's modulus and high actuator strain. For the hole doping $(q>0)$, the maximum $W_{\mathrm{s}}$ is found in the $\mathrm{WTe}_{2}\left(16.4 \mathrm{MJ} \mathrm{m}^{-3}\right.$ at $0.1 \mathrm{e} /$ atom $)$ and $\mathrm{WS}_{2}$ monolayers (7.03 $\mathrm{MJ} \mathrm{m}{ }^{-3}$ at $0.06 \mathrm{e} /$ atom) for the $1 \mathrm{H}$ and $1 \mathrm{~T}$ structures, respectively. $W_{\mathrm{s}}$ at the electron doping case is thus higher than that of the hole doping case, which suggests that the electron doping should be good to achieve high-performance actuator in the artificial muscle application. All the periodic trends strictly obeyed by our data are summarized for the electron doping at $q$ $=-0.1 \mathrm{e} /$ atom are shown in Fig. 8 .

\section{Conclusion}

In summary, we have investigated the actuator performance of the two-dimensional $\mathrm{MX}_{2}$ monolayers with the $1 \mathrm{H}$ and $1 \mathrm{~T}$ structures by first principles calculations. We find that the actuator performance of the $\mathrm{MX}_{2}$ monolayers not only depend on the charge doping level but also the atomic numbers $Z_{\mathrm{M}}$ and $Z_{\mathrm{X}}$ of $\mathrm{M}$ and $\mathrm{X}$ atoms, respectively. The $1 \mathrm{H}-\mathrm{WTe} \mathrm{T}_{2}$ and $1 \mathrm{~T}-\mathrm{WS}_{2}$ monolayers have the best electromechanical performances in the $\mathrm{MX}_{2}$ compounds with the work density per cycle can be achieved up to $41.66 \mathrm{MJ} \mathrm{m}^{-3}$ and $43.30 \mathrm{MJ} \mathrm{m}^{-3}$ under charge doping, respectively. Moreover, the $\mathrm{MX}_{2}$ monolayers show the reversible strain up to $3 \%$. The results of this study are useful for the design and fabricating of artificial muscles with the $\mathrm{MX}_{2}$ compounds.

\section{Conflicts of interest}

There are no conflicts to declare.

\section{Acknowledgements}

This research is funded by the Hanoi University of Science and Technology (HUST) under project number T2017-LN-10.

\section{References}

1 C. Lee, X. Wei, J. W. Kysar and J. Hone, Science, 2008, 321, 385-388.

2 K. S. Novoselov, V. Fal, L. Colombo, P. Gellert, M. Schwab, K. Kim, et al., Nature, 2012, 490, 192.

3 K. Hu, D. D. Kulkarni, I. Choi and V. V. Tsukruk, Prog. Polym. Sci., 2014, 39, 1934-1972.

4 A. K. Geim, Science, 2009, 324, 1530-1534.

5 S. Park, J. An, J. W. Suk and R. S. Ruoff, Small, 2010, 6, 210212.

6 X. Xie, H. Bai, G. Shi and L. Qu, J. Mater. Chem., 2011, 21, 2057-2059.

7 Y. Ge, R. Cao, S. Ye, Z. Chen, Z. Zhu, Y. Tu, D. Ge and X. Yang, Chem. Commun., 2018, 54, 3126-3129. 
8 C. Lu, Y. Yang, J. Wang, R. Fu, X. Zhao, L. Zhao, Y. Ming, Y. Hu, H. Lin, X. Tao, et al., Nat. Commun., 2018, 9, 752.

9 Y. Ding, Y. Wang, J. Ni, L. Shi, S. Shi and W. Tang, Physica B Condens. Matter., 2011, 406, 2254-2260.

10 A. Ostadhossein, A. Rahnamoun, Y. Wang, P. Zhao, S. Zhang, V. H. Crespi and A. C. Van Duin, J. Phys. Chem. Lett., 2017, 8, 631-640.

11 M. Acerce, E. K. Akdoğan and M. Chhowalla, Nature, 2017, 549,370 .

12 N. T. Hung, A. R. Nugraha and R. Saito, J. Phys. D: Appl. Phys., 2018, 51, 075306.

13 H. Ramakrishna Matte, A. Gomathi, A. K. Manna, D. J. Late, R. Datta, S. K. Pati and C. Rao, Angew. Chem., Int. Ed., 2010, 49, 4059-4062.

14 J. N. Coleman, M. Lotya, A. ONeill, S. D. Bergin, P. J. King, U. Khan, K. Young, A. Gaucher, S. De, R. J. Smith, et al., Science, 2011, 331, 568-571.

15 Z. Zeng, Z. Yin, X. Huang, H. Li, Q. He, G. Lu, F. Boey and H. Zhang, Angew. Chem., 2011, 123, 11289-11293.

16 A. Castellanos-Gomez, M. Barkelid, A. Goossens, V. E. Calado, H. S. van der Zant and G. A. Steele, Nano Lett., 2012, 12, 3187-3192.

17 P. Giannozzi, S. Baroni, N. Bonini, M. Calandra, R. Car, C. Cavazzoni, D. Ceresoli, G. L. Chiarotti, M. Cococcioni, I. Dabo, et al., J. Phys.: Condens. Matter, 2009, 21, 395502.

18 K. Lejaeghere, G. Bihlmayer, T. Björkman, P. Blaha, S. Blügel, V. Blum, D. Caliste, I. E. Castelli, S. J. Clark, A. Dal Corso, et al., Science, 2016, 351, aad3000.

19 J. P. Perdew, K. Burke and M. Ernzerhof, Phys. Rev. Lett., 1996, 77, 3865.

20 H. J. Monkhorst and J. D. Pack, Phys. Rev. B: Condens. Matter Mater. Phys., 1976, 13, 5188.

21 C. G. Broyden, IMA Journal of Applied Mathematics, 1970, 6, 222-231.

22 R. Fletcher, Comput. J., 1970, 13, 317-322.
23 D. Goldfarb, Math. Comput., 1970, 24, 23-26.

24 D. F. Shanno, Math. Comput., 1970, 24, 647-656.

25 N. T. Hung, A. R. Nugraha and R. Saito, Carbon, 2017, 118, 278-284.

26 N. T. Hung, A. R. Nugraha and R. Saito, Carbon, 2017, 125, 472-479.

27 A. Dal Corso, J. Phys.: Condens. Matter, 2016, 28, 075401.

28 X. Sun, Z. Wang, Z. Li and Y. Q. Fu, Sci. Rep., 2016, 6, 26666. 29 N. Savjani, E. A. Lewis, M. A. Bissett, J. R. Brent, R. A. Dryfe, S. J. Haigh and P. OBrien, Chem. Mater., 2016, 28, 657-664. 30 Q. D. Truong, N. T. Hung, Y. Nakayasu, K. Nayuki, Y. Sasaki, D. Murukanahally Kempaiah, L.-C. Yin, T. Tomai, R. Saito and I. Honma, RSC Adv., 2018, 8, 33391-33397.

31 E. Torun, H. Sahin, S. Cahangirov, A. Rubio and F. M. Peeters, J. Appl. Phys., 2016, 074307.

32 Y. Du, J. Maassen, W. Wu, Z. Luo, X. Xu and P. D. Ye, Nano Lett., 2016, 16, 6701-6708.

33 J.-W. Jiang and H. S. Park, Nano Lett., 2016, 16, 2657-2662. 34 H. Wang, X. Li, P. Li and J. Yang, Nanoscale, 2017, 9, 850-855. 35 R. H. Baughman, J. M. Shacklette, A. A. Zakhidov and S. Stafström, Nature, 1998, 392, 362.

36 F. Mouhat and F. Coudert, Phys. Rev. B: Condens. Matter Mater. Phys., 2014, 90, 224104.

37 J. Y. Rho, R. B. Ashman and C. H. Turner, J. Biomech., 1993, 26, 111-119.

38 G. W. Rogers and J. Z. Liu, J. Am. Chem. Soc., 2011, 133, 10858-10863.

39 J. D. Madden, N. A. Vandesteeg, P. A. Anquetil, P. G. Madden, A. Takshi, R. Z. Pytel, S. R. Lafontaine, P. A. Wieringa and I. W. Hunter, IEEE J. Oceanic Eng., 2004, 29, 706-728.

40 J. Weissmüller, R. Viswanath, D. Kramer, P. Zimmer, R. Würschum and H. Gleiter, Science, 2003, 300, 312-315.

41 H.-J. Jin, X.-L. Wang, S. Parida, K. Wang, M. Seo and J. Weissmuller, Nano Lett., 2009, 10, 187-194. 